\title{
Dietary intake of trans fatty acids as a cardiovascular risk factor in a population of Italian teenagers
}

\author{
Francesco Sofi, ${ }^{1,2,3}$ Daniele Rapini, ${ }^{1}$ Giulia Innocenti, ${ }^{1}$ Rosanna Abbate, ${ }^{2}$ Gian Franco Gensini, ${ }^{2,3}$ \\ Alessandro Casini ${ }^{1}$ \\ ${ }^{1}$ Regional Agency of Nutrition, Azienda Ospedaliero-Universitaria Careggi, University of Florence, Italy; \\ ${ }^{2}$ Department of Medical and Surgical Critical Care, Thrombosis Centre, University of Florence; ${ }^{3}$ Don Carlo Gnocchi \\ Foundation, Onlus IRCCS, Impruneta, Florence, Italy
}

\begin{abstract}
Trans fatty acids are unsaturated fatty acids produced by the partial hydrogenation of polyunsaturated oils. Over the last few years, an increasing interest on these fatty acids has been shown because of their role in the pathogenesis of cardiovascular diseases. To date, major scientific associations strongly recommend consuming a low intake of trans fatty acids for the prevention of cardiovascular diseases, but data on the consumption of these fatty acids in the general population are still lacking. We conducted this observational study on a population of Italian teenagers in order to evaluate the consumption of trans fatty acids in the diet. We studied 81 Italian teenagers, 45 males and 36 females, with a median age of 16 years. To assess their consumption of trans fatty acids, we used the "High School Survey", a questionnaire prepared by the Harvard Medical School. Total calories of the studied population were $2359.2 \pm 591.5 \mathrm{kcal} /$ day with a mean intake of trans fatty acids of $3.24 \pm 1.48 \mathrm{~g} /$ day, corresponding to $1.23 \%$ of the total energy. A relevant proportion of subjects, namely $51(62.9 \%)$, exceeded the limit contribution of $1 \%$ of energy from trans fatty acids. Their intake of total calories and trans fatty acids significantly increased according to their increasing age $(p=0.0003$ for trend). Our data, therefore, obtained in a limited population of Italian adolescents, showed that a consistent proportion of adolescents does not follow the nutritional recommendations for intake of trans fatty acids, likely increasing their risk of cardiovascular diseases.
\end{abstract}

Keywords: Nutrition; adolescents; risk factors; diet

Received: 30 March 2009; Accepted: 19 July 2009; First published online: 14 October 2009

$\mathrm{T}$ RANS FATTY ACIDS ARE FATTY ACIDS PRODUCED BY the partial hydrogenation of polyunsaturated oils. ${ }^{1}$ Margarine, baking products, fried food and dairy products represent the most important dietary sources of these particular types of fatty acids. $^{2}$ Over the last years, an increasing amount of studies reported the relevant role that the acids have on increasing the cardiovascular risk of the general population. ${ }^{3-5}$ It has been demonstrated that the

Correspondence to: Francesco Sofi, MD, PhD, Department of Medical and Surgical Critical Care, Thrombosis Centre, University of Florence, Italy, Viale Morgagni, 85, 50134 Florence, Italy. Tel: +39-055-7949420; Fax: +39-0557949418; E-mail: francescosofi@gmail.com acids are able to determine a significant increase of the low density lipoprotein-cholesterol, and, at the same time, a decrease of the high density lipoprotein-cholesterol. ${ }^{5}$ Furthermore, their unfavourable effect on atherosclerosis has been ascribed to their effects on the inflammatory pathway, through the increase of the concentration of some inflammatory cytokines. ${ }^{6}$ Accordingly, these two aspects reflect a great raise of the relative risk of coronary arterial disease in the quartile of population with a higher introduction of trans fatty acids, and it has been also demonstrated that their capacity to modify the normal evolution of the coronary arterial disease is more dramatic than that produced 
by saturated fatty acids. ${ }^{7}$ In addition, the trans fatty acids have also been reported to increase the insulin resistance in subjects with diabetes mellitus type 2 and obesity, ${ }^{8}$ influencing the development of the Alzheimer's disease 9 by inhibiting the activity of $\Delta 5$ and $\Delta 6$ desaturase, and causing a reduction of synthesis of long-chain polyunsatured fatty acids, which are part of the neuronal structures. Recently, due to all these negative effects of trans fatty acids, guidelines and recommendations with the aim of lowering the intake of these particular fatty acids, have been released by the most important scientific societies. The Dietary Guidelines for Americans in 2005 reported that their consumption should be kept "as low as possible", ${ }^{10}$ or "kept at a low intake", while the American Heart Association recently suggested a maximum safe intake of $1 \%$ of energy deriving from trans fatty acids. ${ }^{11}$ According to these scientific publications, the government of the United States of America published a law with the aim of reducing the consumption of trans fatty acids. This action was also taken by the Danish government, which published a "Trans Fat Ban". ${ }^{12}$ The situation in Italy, in contrast, is far from the American and the Danish experiences. The only publication with the aim of defining the safest intake of trans fatty acids is represented by the Livelli di Assunzione Raccomandati di Energia e Nutrienti per la Popolazione Italiana, a technical book published in 1997. ${ }^{13}$ To the best of our knowledge, no studies trying to obtain data on the consumption of trans fatty acids by the Italian population have been published. Because of this, we conducted this observational study on a population of Italian teenagers in order to evaluate the consumption of trans fatty acids, and possibly promote a discussion towards the presence of these fatty acids in the Italian diet.

\section{Material and Methods}

\section{Study design and Participants}

We conducted an observational study in a population of healthy teenagers recruited at the high school Liceo Scientifico Francesco Redi, in Arezzo, Italy. Recruitment took place in November of 2007. The population was composed by 81 individuals, with 23 drawn from the 2 nd class, 21 from the 3 rd, 17 from the 4th, and 20 from the 5th class.

\section{Food frequency assessment}

In order to assess the consumption of trans fatty acids, we used the High School Survey, a questionnaire published by the Harvard Medical School, and used within the Nurses' Health Study. The choice of a questionnaire designed for use in the United States of America was predicated by the lack of a specific questionnaire in the Italian scientific literature, as well as by the similarity of our population with the one analysed by the Harvard Medical School. In addition to the original questionnaire, we added a preface describing the objectives of the study, and a panel with the number of identification of the student and some information. The questionnaire was self-administered in the classrooms during school time in presence of an examiner ready to answer every question or problem that occurred during the compilation. To avoid some inaccuracies in the identification of the portions, we used a photographic atlas of food.

\section{Analysis and calculation of the food frequency questionnaires}

After the completion of quetionnaires, we analysed the answers given using the software WinFood Due (Medimatica Srl, Colonnella, TE, Italy). Due to the lack of trans fatty acids within the Italian composition tables, we inserted the values reported in 3 different tables, specifically the Danish food composition databan" published by The Technical University of Denmark, the Norwegian food composition table 200" of the Norwegian Food safety Authority, and the Department of Agriculture of the United States of America Fat and Fatty acid Content of Selected Foods Containing Trans-Fatty Acids.

\section{Statistical analysis}

Statistical analysis was performed by using the SPSS (Chicago, IL, USA) software for Windows (Version 15.0). Continuous variables were expressed as mean plus or minus standard deviations for parametric data, or median and range for non-parametric data. The non-parametric Mann-Whitney test was used for comparison between single groups. The KruskalWallis test was used for comparison among different groups, and the Chi-square test was used to test for comparison between proportions. Correlation analyses were performed by Spearman's correlation test. A p value lower than 0.05 was considered to be statistically significant.

\section{Results}

Our cohort comprised 81 adolescents, 45 males and 36 females, with a median age of 16 years. Total calories of the general population were $2359.2 \pm$ 591.5, with a percentage caloric intake of macronutrients of $53.1 \pm 4.6 \%$ for carbohydrates, $14.0 \pm 1.4 \%$ for protein, and $32.8 \pm 4.4 \%$ for total fats. Dietary characteristics of the whole population of teenagers according to gender are reported in Table 1. No significant differences were observed for all the variables considered. A significant correlation 
Table 1. Dietary pattern of the cohort.

\begin{tabular}{lccc}
\hline Variables & Males $(\mathrm{n}=45)$ & Females $(\mathbf{n}=36)$ & p value \\
\hline Total calories (Kcal/day) & $2490.1 \pm 560.5$ & $2195.5 \pm 596$ & 0.09 \\
Carbohydrates (\% energy) & $52.9 \pm 4.9$ & $53.4 \pm 4.3$ & 0.6 \\
Protein (\% energy) & $14.1 \pm 1.4$ & $13.9 \pm 1.3$ & 0.9 \\
Total fats (\% energy) & $32.9 \pm 4.6$ & $32.7 \pm 4.2$ & 0.9 \\
Trans fatty acids (\% energy) & $1.23 \pm 0.41$ & $1.23 \pm 0.47$ & 0.8 \\
\hline
\end{tabular}

All data are presented as mean \pm standard deviation.

Table 2. Dietary characteristics according to the classes.

\begin{tabular}{lcccc}
\hline Variables & 2nd class & 3rd class & 4th class & 5th class \\
\hline Total calories (Kcal/day) & $2040.2 \pm 435.4$ & $2480.4 \pm 672.2$ & $2434.5 \pm 520.2$ & $2534.8 \pm 611.1 *$ \\
Carbohydrates (\% energy) & $51.6 \pm 4.1$ & $53.8 \pm 4.6$ & $54.5 \pm 4.5$ & $52.9 \pm 5.2$ \\
Protein (\% energy) & $14.4 \pm 1.4$ & $13.9 \pm 1.1$ & $13.6 \pm 1.4$ & $14.1 \pm 1.5$ \\
Total fats (\% energy) & $33.9 \pm 3.8$ & $32.3 \pm 4.0$ & $31.9 \pm 4.3$ & $32.9 \pm 5.4$ \\
Trans fatty acids (\% energy) & $1.13 \pm 0.40$ & $1.17 \pm 0.33$ & $1.52 \pm 0.50$ & $1.16 \pm 0.42 *$ \\
\hline
\end{tabular}

All data are presented as mean \pm standard deviation.

*p lower than 0.05 for trend.

between total lipid intake and trans fatty acids was evident for the whole population, with a $\mathrm{R}$ of 0.25 and $\mathrm{p}$ of 0.02 . With regard to trans fatty acids, in the whole population we observed an intake of $3.24 \pm$ 1.48 grams per day, corresponding at $1.23 \pm 0.44 \%$ of the total daily calories. A majority of subjects, namely 51 (62.9\%), exceeded the limit contribution of $1 \%$ of the total calories obtained by trans fatty acids as recommended by the American Heart Association. In order to test the possible relationship between consumption and age, we conducted an analysis of dietary pattern through the different classes of the teenagers. Total calories and contribution from the trans fatty acids significantly increased according to the classes (Table 2), with a significant increasing number of teenagers that exceeded the limit value of $1 \%$ of trans fatty acids according to the increasing class number (Fig. 1). We finally performed a general linear model adjusted for total intake of fats, confirming the significant increasing values of trans fatty acids according to the different classes ( $\mathrm{p}=0.003$ for trend).

\section{Discussion}

Our study was performed to assess dietary habits of a limited population of Italian teenagers in relation to the consumption of trans fatty acids. Our data, obtained in a typical population of young Mediterranean adolescents, showed that over than half of the population exceeded the value of $1 \%$ of total calories obtained through this particular type of fatty acids. This datum is extremely important, not

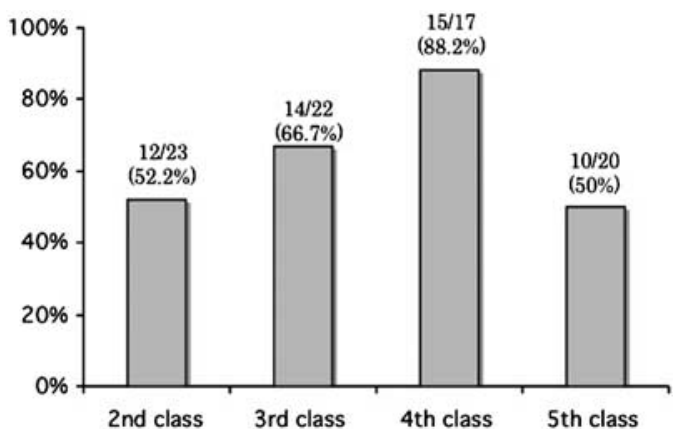

Figure 1.

Subjects whose contribution of trans fatty acids exceeded the limit value of $1 \%$ of total calories according to classes.

only for the considerations it brings relative to health, but also because it shows the obsolescence of the Italian laws on this issue. To date, in Italy there are no specific laws for regulation of trans fatty acids in the foods.

Trans fatty acids are unsaturated fatty acids with at least one double bond in the trans configuration. ${ }^{1}$ Over the last decades, there has been increasing interest in this type of fatty acids because of its supposed hazardous effects on lipid metabolism, so increasing the risk of cardiovascular diseases. A recent meta-analysis of 13 randomized controlled trials indicated that isocaloric replacement of either saturated or cis unsaturated fats with trans fatty acids raises low-density lipoprotein-cholesterol and reduces high-density lipoprotein-cholesterol, ${ }^{14}$ and prospective studies investigating the associations between trans fatty acids and coronary arterial 
disease demonstrate an incidence of disease attributable to the intake of trans fatty acids that is greater than that predicted by changes in serum lipids alone. ${ }^{1}$ Furthermore, trans fatty acids have other unfavourable effects on cardiovascular health, ranging from the inflammatory pathway to endothelial dysfunction. ${ }^{6}$ Cardiovascular diseases represent the first cause of death in the industrialized countries, and it has been estimated that a detailed law with the aim of reducing the intake of trans fatty acids over the general population would prevent between 600 and 1800 heart attacks, saving 250-500 people and 13 years of life per head, with an economical gain of 26,757 million dollars. Some experiences have been conducted worldwide to reduce the intake of trans fatty acids. In Denmark, after a period of 30 years monitoring their content in Danish foods, made by the Danish Institute for Food and Veterinary Research, the Danish Order no. 160 of March 11, 2003 imposed a maximum of 2\% of trans fatty acids in oils and fats destined for human consumption. ${ }^{15}$ As a result, the amount of industrially-produced trans fatty acids has significantly reduced, stimulating producers to develop new methods of production without increasing prices or reducing variability in the products. Likewise, as of December 12, 2005 in Canada, ${ }_{17}^{16}$ and January 1, 2006, in the United States, ${ }^{17}$ nutrition labels for all conventional foods and supplements were required to list the content of trans fatty acids. These labelling changes, together with publicity surrounding harmful effects of trans fatty acids, prompted subjects to consume a level "as low as possible" of trans fatty acids with the foods, and some food manufacturers to reformulate their products to reduce or eliminate trans fatty acids. Recently, following the lead of Denmark, New York City has passed legislation to reduce the use of trans fatty acids in food service establishments. ${ }^{18}$ In fact, as of July 1st, 2007 in New York City, food service establishments can no longer fry with fats that contain more than $0.5 \mathrm{~g}$ of trans fatty acids per serving, and as of July 1st, 2008, serve foods that contain more than $0.5 \mathrm{~g}$ of trans fatty acids per serving. In industrialized countries, major dietary sources of trans fatty acids include bakery products, deep fried and frozen foods, packaged snacks, margarine, and crackers. In Italy, conversely, to the best of our knowledge, no acts or rules trying to regulate or minimize the presence of trans fatty acids in foods are available. The last update of Italian tables for food composition, made in 1996, indicate a daily intake of 5 grams of trans fatty acids, which is much higher than that declared by the other governments or scientific societies. No data on the consumption of trans fatty acids in the
Italian population is available. $^{13}$ The present, although limited, is the first analysis of consumption of trans fatty acids in an Italian population. We decided to study the dietary profile of a subset of Italian teenagers in order to estimate the cardiovascular risk profile of this particular portion of the general population, as a mirror of the future adult population. The comparison between our results and the latest scientific publications showed that the value observed in the whole population is higher than the most updated reference values. Moreover, with regard to the maximum level recommended by the American Hearth Association in 2006, ${ }^{19}$ over than half of our population of teenagers exceeded this limit, by significantly increasing their risk of cardiovascular diseases. The importance of diet on the health of young adults has been intensively reported over the last years and a strict relationship between unhealthy choices of food and disease states has been demonstrated in adolescents, showing that primary prevention is extremely important in such a population. ${ }^{20}$

Our study has several limitations. First of all, it is a preliminary investigation, with a very limited number of subjects. This does not allow us to give any reliable conclusion that can be extended to the general Italian population. Nevertheless, it is the first available study that investigated this issue in the Italian population. Second, due to the lack of information regarding the content of trans fatty acids in the Italian foods, we could not calculate their values by using Italian food composition tables, being forced to use food tables from different countries. Third, we could not estimate the reproducibility of the questionnaire used in our cohort.

In conclusion, our data, although preliminary and performed in a very limited number of subjects, showed that dietary contribution of trans fatty acids in the diet of the Italian teenagers is higher than recommended by the major scientific association for a optimal prevention of cardiovascular diseases.

\section{References}

1. Micha R, Mozaffarian D. Trans fatty acids: effects on cardiometabolic health and implications for policy. Prost Leukotriens Fatty Acids 2008; 79: 147-152.

2. Mozaffarian D, Willett WC. Trans fatty acids and cardiovascular risk: a unique cardiometabolic imprint? Curr Atheroscler Rep 2007; 9: 486-493.

3. Lichtenstein AH. Trans Fatty Acids, Plasma Lipid Levels, and Risk of Developing Cardiovascular Disease. Circulation 1997; 95: 2588-2590.

4. Booker CS, Mann JI. Trans fatty acids and cardiovascular health: translation of the evidence base. Nutr Metab Cardiovasc Dis 2008; 18: 448-456.

5. Sun Q, Ma J, Campos $\mathrm{H}$, et al. A prospective study of trans fatty acids in erythrocytes and risk of coronary heart disease. Circulation 2007; 115: 1858-1865. 
6. Mozaffarian D. Trans Fatty Acids- effects on systemic inflammation and endothelial function. Atherosclerosis Suppl 2006; 7: 29-32.

7. Mozaffarian D, Katan MB, Ascherio A, Stampfer MJ, Willett WC. Trans fatty acids and cardiovascular diseases. N Engl J Med 2006; 354: 1858-1865.

8. Bray GA, Lovejoy JC, Smith SR, et al. The influence of different fats and fatty acids on obesity, insulin resistance and inflammation. J Nutr 2002; 132: 2488-2491.

9. Morris MC, Evans DA, Bienias JL, et al. Dietary fats and the risk of incident Alzheimer disease. Arch Neurol 2003; 60: 194-200.

10. US Department of Agriculture. Dietary Guidelines for Americans, 2005.

11. Third report of the National Cholesterol Education Program (NCEP) expert panel on detection, evaluation, treatment of high blood cholesterol in adult (Adult Treatment Panel III). Final Report. Circulation 2002; 106: 3143-3421.

12. Astrup A. The trans fatty acid story in Denmark. Atherosclerosis Suppl 2006; 7: 43-46.

13. LARN, Composizione degli alimenti, aggiornamento 2000. Edizioni EDRA. Available at: http://www.sinu.it/larn

14. Mozaffarian D, Clarke R. Quantitative effects on cardiovascular risk factors and coronary heart disease risk of replacing partially hydrogenated vegetable oils with other fats and oils. Eur J Clin Nutr (in press).

15. Leth T, Jensen HG, Mikkelsen AE, Bysted A. The effect of the regulation on trans fatty acid content in Danish food. Atherosclerosis Suppl 2006; 7: 53-56.
16. Health Canada. Government response to the interim recommendations of the trans fat task force 2005. Available from: http:// www.hc-sc.gc.ca/fn-an/nutrition/gras-trans-fats/government_response_ reponse_gouvernement_e.html

17. Food and drug administration, Food labeling: Trans fatty acids in nutrition labeling, nutrient content claims, and health claims, in Federal Register, Department of Health and Human Services, Editor 2003.

18. New York City Department of Health and Mental Hygiene, press release: $94 \%$ of inspected restaurants in compliance with first phase of trans fat regulation 2007. Available from: http:// www.nyc.gov/html/doh/html/pr2007/pr080-07.shtml

19. Lichtenstein AH, Appel LJ, Brands M, et al. Diet and lifestyle recommendations Revision 2006. A scientific statement from the American Heart Association Nutrition Committee. Circulation 2006; 114: 82-96.

20. Steinberger J, Daniels SR, Eckel RH, et al. American Heart Association Atherosclerosis, Hypertension, and Obesity in the Young Committee of the Council on Cardiovascular Disease in the Young; Council on Cardiovascular Nursing; and Council on Nutrition, Physical Activity, and Metabolism. Progress and challenges in metabolic syndrome in children and adolescents: a scientific statement from the American Heart Association Atherosclerosis, Hypertension, and Obesity in the Young Committee of the Council on Cardiovascular Disease in the Young; Council on Cardiovascular Nursing; and Council on Nutrition, Physical Activity, and Metabolism. Circulation 2009; 119: 628-647. 